Research Article

\title{
Neural Network Algorithm-Based Three-Dimensional Ultrasound Evaluation in the Diagnosis of Fetal Spina Bifida
}

\author{
Lei Chen $\mathbb{D}^{1},{ }^{1}$ Yingying Tian $\mathbb{D}^{2},{ }^{2}$ and Yujie Deng $\mathbb{D}^{2}$ \\ ${ }^{1}$ Department of Ultrasound, The Affiliated Suzhou Hospital of Nanjing Medical University, Suzhou 215008, Jiangsu, China \\ ${ }^{2}$ Department of Ultrasound, The Affiliated Suzhou Science \& Technology Town Hospital of Nanjing Medical University, \\ Suzhou 215000, Jiangsu, China \\ Correspondence should be addressed to Yujie Deng; 3090102312@caa.edu.cn
}

Received 13 May 2021; Revised 12 July 2021; Accepted 26 July 2021; Published 15 August 2021

Academic Editor: Enas Abdulhay

Copyright (C) 2021 Lei Chen et al. This is an open access article distributed under the Creative Commons Attribution License, which permits unrestricted use, distribution, and reproduction in any medium, provided the original work is properly cited.

In order to realize the automatic recognition and diagnosis in ultrasound images of fetal spina bifida, the U-Net algorithm was improved in this study to obtain a new convolutional neural network algorithm-Oct-U-Net. 3,300 pregnant women were selected as the research objects, who underwent three-dimensional (3D) ultrasound examinations. Then, Oct-U-Net was applied to evaluate the diagnostic effect of fetal spina bifida by recall rate, precise rate, mean standard error, pixel accuracy (PA), mean intersection over union (MIoU), and running time. Besides, the fully convolutional network (FCN) algorithm and the U-Net algorithm were introduced for comparison. Results showed that recall rate, precise rate, PA, and MioU of Oct-U-Net were $0.93,0.96,0.949$, and 0.917 , respectively, which were markedly higher than those of FCN and U-Net $(P<0.05)$. The mean standard error of Oct-U-Net was 4.1243, and its average running time was 12.15 seconds. The values of the above two indicators were sharply lower than those of FCN and U-Net $(P<0.05)$. In conclusion, Oct-U-Net had a better diagnostic effect on 3D ultrasound images of fetal spina bifida, with higher segmentation accuracy and shorter running time, so it was worthy of clinical application.

\section{Introduction}

Fetal spina bifida is a common fetal congenital malformation, with a clinical incidence of about $0.1 \%$, which is one of the main causes of neonatal death and disability [1]. Fetal spina bifida is that the fetal neural tube is damaged when it is closed due to some physical and chemical factors during the early pregnancy and the embryonic development of the fetus, resulting in incomplete closure of the fetal spinal canal and cracks [2,3]. Furthermore, there are two types of fetal spina bifida, including open and closed. In closed fetal spina bifida, there is only a defect in the fetal spinal canal, no content and meningocele, and the continuous intact of the skin at the fetal lesion. There are no obvious symptoms during the examination, and the clinical detection rate is low [4]. Open spina bifida will cause bulging of the contents of the fetal spinal canal, and the continuous interruption can be often observed in the skin of the lesion, often accompanied by back masses and cerebrospinal fluid exudation from the defect [5]. The survival rate of fetuses with open spina bifida is extremely low after birth, and even if they can survive, they are often accompanied by lifelong disabilities. Therefore, most pregnant women choose to receive diagnosis and treatment as soon as possible, terminate the pregnancy, and abandon the birth of a spina bifida fetus $[6,7]$.

At present, there are two main clinical examination methods for fetal spina bifida, which are prenatal biochemical examination and prenatal ultrasound examination. Prenatal biochemical examination is mainly an examination method for open fetal spina bifida. The biochemical examination indicators include pregnant women's amniotic fluid alpha-fetoprotein (AFP), amniotic fluid acetylcholinesterase (AChE), and maternal serum alpha-fetoprotein (MS-AFP) $[8,9]$. Moreover, ultrasound examination is the main method of prenatal examination for pregnant women, which can diagnose open fetal spina bifida and partially closed fetal spina bifida. During the ultrasound examination, the detection rate of fetal spina bifida is closely related to the 
operating experience of clinicians, fetal position, AFP, AchE, and MS-AFP [10]. 3D ultrasound deals with the feature that traditional 2D ultrasound can only obtain single profile information so as to obtain the $3 \mathrm{D}$ structure of the patient through mechanical scanning, free-arm scanning, or $2 \mathrm{D}$ array scanning [11]. 3D ultrasound examination is convenient, noninvasive, and economical and will not cause adverse effects on the mother and fetus. It is an indispensable detection method in the prenatal examination of the mother [12].

With the development of computer technology, computer-aided diagnosis (CAD) technology has been increasingly applied in the medical industry, especially with the development of deep learning and convolutional neural networks; it has brought great convenience to clinicians' image diagnosis [13]. Cengizler et al. [14] believed that a computerized support system that automatically detected the position of spinal deformity might help clinicians diagnose spina bifida, but an algorithm was needed to identify the entire spine in ultrasound images. However, ultrasound images often contain a large number of noise spots in actual clinical diagnosis due to its inherent features. These noises lead to great difficulty in image processing and image-based follow-up diagnosis, treatment, and quantitative analysis $[15,16]$. With the development of neural networks, many algorithms for feature extraction of ultrasound images have been proposed, and good results have been achieved in the field of medical image processing, but there is still a gap in ultrasound image processing of fetal spina bifida. On this basis, this research improved the U-Net algorithm, introduced an Octave feature to reduce redundant information, proposed a new neural network algorithm-Oct-U-Net, and implemented ultrasound for fetal spina bifida image lesion diagnosis and accurate segmentation; this research aimed to provide a theoretical basis for the clinical diagnosis of fetal spina bifida.

\section{Materials and Methods}

2.1. Research Objects. A total of 3,300 pregnant women, who underwent $3 \mathrm{D}$ ultrasound examinations in hospital from November 2015 to November 2020, were selected as the research objects. They were $21-36$ years old, with an average age of 26.4 years, and the gestational age was $23-31$ weeks, with an average gestational age of 24.5 weeks. Besides, the fetal head-hip diameter is $46-82 \mathrm{~mm}$. This experiment had been approved by the Medical Ethics Committee of hospital. All pregnant women and their family members had understood this experiment and signed the informed consent forms.

The criteria for inclusion were defined to include patients who had the gestational age of 17-38 weeks with the fetal head-hip diameter of 45-84 $\mathrm{mm}$, were single or twin, and had the complete clinical data.

The criteria for exclusion were defined to include patients who had three or more multiple pregnancies, refused to undergo 3D ultrasound examinations, had incomplete clinical data, and were not suitable for this experiment for other reasons.
2.2. Ultrasound Examination Parameters. All the pregnant women were in the supine position during examinations. A GE Voluson E8 color Doppler ultrasound diagnostic apparatus and a $3 \mathrm{D}$ volume probe of the abdomen were applied to this experiment. The frequency was adjusted to 2-6 MHz, and the 6-D probe was selected. Moreover, the abdomen of each pregnant woman was detected by continuous sagittal, transverse, and coronal examination. All ultrasound examinations were carried out in strict accordance with the examination guidelines. The fetal head, posterior cervical fossa, and spine were scanned and detected, and the curvature and continuity of the fetal spine and covering skin were observed so as to record relevant data. If a suspected spinal disease was detected, a high-frequency probe could be employed to further investigate the location of the medullary cone. After the examination, the ultrasound image was saved in the workstation.

\subsection{Implementation of Oct-U-Net Neural Network Algorithm.} Convolutional neural network is a good image processing method. As a special neural network, it includes a data input layer and a convolutional layer. The convolutional layer is composed of convolution operation, activation function, and pooling [17]. In addition, the specific structure is shown in Figure 1.

A set of raw data was input into the convolutional neural network, and the data obtained after convolution of the original data and the convolution kernel were more featuremapped. For a neural network, its calculation equation on a 2D tensor could be expressed as follows:

$$
y[m, n]=\sum_{a} \sum_{b} x[m+a, n+b] w[a, b] .
$$

In equation ( 1$), y[m, n]$ represents the output $2 \mathrm{D}$ tensor, $x[m, n]$ stands for the input $2 \mathrm{D}$ tensor, and $w[a, b]$ expresses the weight of the convolution kernel. For a complete convolutional neural network, each layer contains several feature images. Therefore, it was assumed that each layer contained $n$ convolution kernels and each convolution kernel consisted of $a$ set of parameters, and the operation of each convolution layer could be expressed as follows:

$$
y_{n}^{l}=\sum_{a=1}^{A} w_{n a}^{l} * y_{a}^{l-1}+c_{n}^{l} .
$$

In equation (2), $y_{n}^{l}$ represents the $n$-th feature image of the $l$-th convolution layer, $w_{n a}^{l}$ stands for the convolution kernel parameter of the $n$-th feature image in the $l$-th convolution layer mapped by the $a$-th feature image of the $l$-1-th convolution layer, $y_{a}^{l-1}$ expresses the $a$-th feature image of the $l-1$-th convolution layer, and $c_{n}^{l}$ means the bias of the $n$-th feature image in the $l$-th convolution layer. Convolution operation is a kind of linear transformation. Generally, a nonlinear activation function needs to be added after each layer of convolution operation in order to realize multilayer nonlinear features. The sigmoid function was 


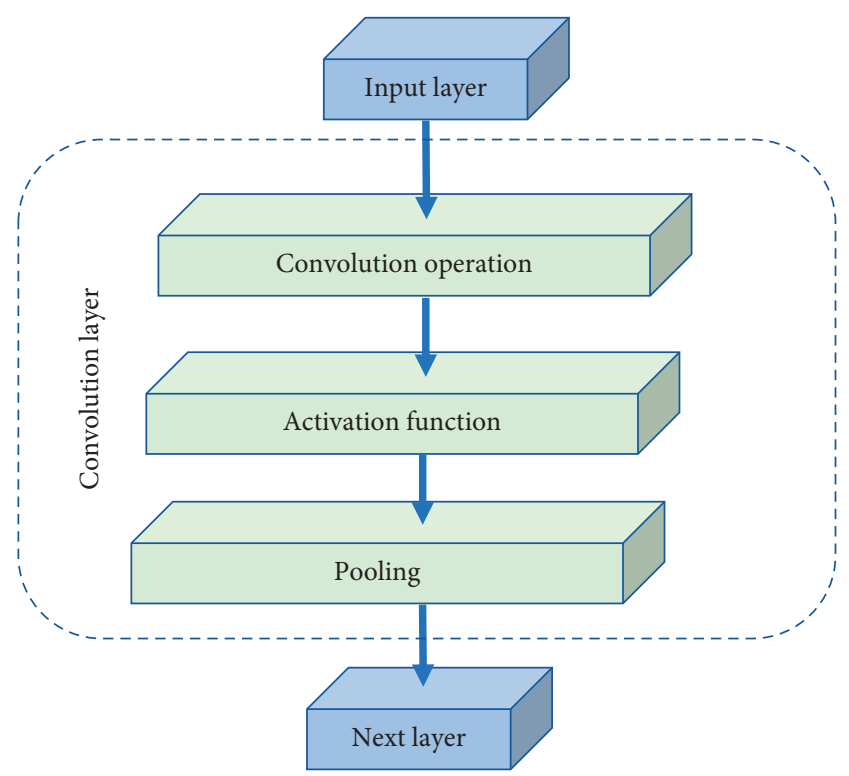

FIGURE 1: Schematic diagram of convolutional neural network structure.

taken as an example, and the expression was presented in the following equation:

$$
f(x)=\frac{1}{e^{-x}+1} .
$$

Pooling operation includes maximum pooling and average pooling. Pooling does not perform weighting operation and does not require activation functions. It simply performs maximum or average operation to express the overall statistical features of the pooling results [18]. Pooling is just to participate in the design of network institutions so as to reduce data dimension, speed up calculation, and prevent overfitting. The pooling operation is represented by Figures 2 and 3.

There are two parts contained in U-Net neural network, namely, feature extraction and upsampling. During feature extraction, each time the image passes through a pooling layer, the image scale will be reduced once. However, each time upsampling will be fused with the channel number of the same scale during feature extraction [19]. U-Net convolution includes two layers of convolution after pooling, which requires a large amount of calculation. In order to reduce the amount of calculation of the U-Net algorithm, the original two-layer convolution can be replaced with one Octave convolution.

When ordinary convolutional neural networks perform calculations, the spatial resolution of the feature images of the input layer and output layer is the same. When capturing image details, it usually contains some spatial redundant information. In order to decrease the redundant information and shorten the calculation time, an Octave feature could be introduced to stand for. Thus, $x$ was denoted as the input tensor of the convolution operation, $n$ was denoted as the number of feature maps, and $a$ and $b$ represented the spatial dimension. The $x$-extended feature image channel could be decomposed into the following:

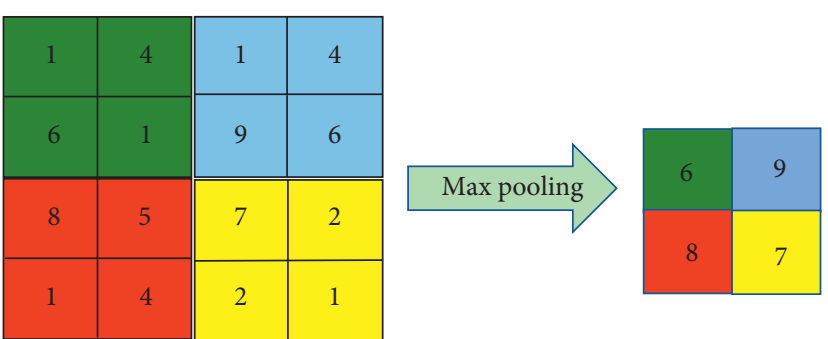

FIgURE 2: Maximum pooling.
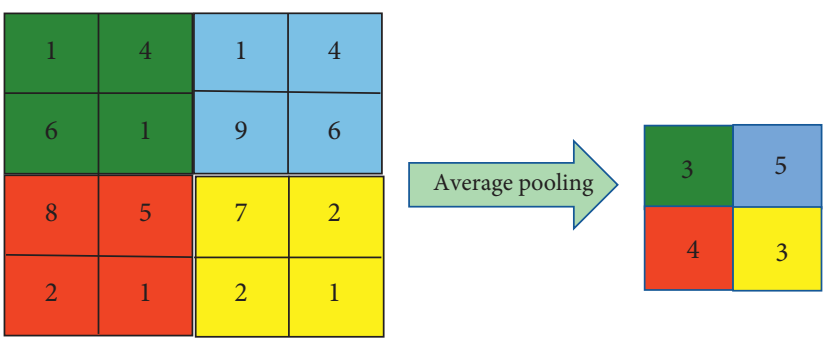

FIgURE 3: Average pooling.

$$
x=\left\{x^{H}, x^{L}\right\} .
$$

In equation (4), $x^{H}$ stands for the captured high-frequency feature map and $x^{L}$ stands for the captured lowfrequency feature map. Then, the expression could be calculated as follows:

$$
\begin{aligned}
& x^{H} \in R^{(1-p) n \times \alpha \times \beta}, \\
& x^{L} \in R^{p n \times((\alpha / 2) \times(\beta / 2))} .
\end{aligned}
$$

In equations (5) and (6), $p$ represents the channel ratio of the low-frequency part. In the Octave convolution operation, the output high-frequency information could be expressed in the following equation:

$$
y=\left\{y^{H}, y^{L}\right\} .
$$

In addition, $y^{H}$ and $y^{L}$ could be presented as follows:

$$
\begin{gathered}
y^{H}=y^{H \longrightarrow H}+y^{L \longrightarrow H}, \\
y^{L}=y^{L \longrightarrow L}+y^{H \longrightarrow L} .
\end{gathered}
$$

In equations (8) and (9), $y^{H \longrightarrow H}$ and $y^{L \longrightarrow L}$ stand for information update within frequency during convolution and $y^{L \longrightarrow H}$ and $y^{H \longrightarrow L}$ mean the information update between frequencies.

In the convolution operation, the convolution kernel $N$ was divided into two components $N^{H}$ and $N^{L}$ to be responsible for the convolution of $x^{H}$ and $x^{L}$, respectively. By further dividing the convolution component into withinfrequency component and out-of-frequency component, the final calculated high-frequency tensor and low-frequency tensor could be expressed as follows: 


$$
y^{H}=f\left(x^{H} ; N^{H \longrightarrow H}\right)+\text { up sample }\left(f\left(x^{L} ; N^{L \longrightarrow H}\right), 2\right),
$$

$$
y^{L}=f\left(x^{L} ; N^{L \longrightarrow L}\right)+f\left(\operatorname{pool}\left(x^{H}, 2\right) ; N^{H \longrightarrow L}\right) .
$$

In equations (10) and (11), $f$ stands for the convolution operation, pool expresses the average pooling with the convolution kernel of $k \times k$ and the step size of $k$, and up sample representes the upsampling by the most recently interpolated factor $k$. Octave convolution can define the lowfrequency and high-frequency space of the convolution operation, thereby reducing spatial redundant information and shortening the calculation amount of the U-Net algorithm.

2.4. Ultrasound Image Diagnosis of Fetal Spina Bifida. 3,300 pregnant women undergoing ultrasound examinations in hospital were detected through 3D ultrasound examinations. Then, 2 ultrasound experts would determine whether the fetus suffered from spina bifida. Pregnant women diagnosed with fetal spina bifida would be treated with labor induction or the fetuses were born in order to confirm the diagnosis, which were subjected to the actual results. Then, Oct-U-Net was applied to analyze the $3 \mathrm{D}$ ultrasound image of each pregnant woman's abdomen to determine whether the fetus had spina bifida. In order to facilitate the comparison of the diagnostic effects of Oct-UNet, FCN and U-Net were introduced for comparative analysis. When applying different algorithms for diagnosis, the recognition was judged as accurate, namely, the algorithm's automatic recognition area and the expert's manual division of the area were basically the same, which were valid positive. Besides, the valid negative could be judged when the fetal spina bifida was recognized by the algorithm and the experts. Based on these two judgment results, the false positive meant that there was spina bifida in fact but it was not detected by the algorithm. The presence of spina bifida detected by the algorithm when there is no spina bifida is considered a false negative. Finally, the precise rate and recall rate were employed to judge the accuracy of different algorithms in the diagnosis of fetal spina bifida:

$$
\begin{gathered}
\text { precise }=\left(\frac{\mathrm{TP}}{\mathrm{TP}+\mathrm{FP}}\right) \times 100 \%, \\
\text { recall }=\left(\frac{\mathrm{TP}}{\mathrm{TP}+\mathrm{FN}}\right) \times 100 \% .
\end{gathered}
$$

In equations (12) and (13), TP, FP, and FN stand for true positive, false negative, and false positive in turn.

2.5. Average Error between the Neural Network Scan Route and the Route Drawn by the Experts. In order to analyze the influence of different neural network algorithms on the segmentation performance of fetal ultrasound images, the segmentation effect of the neural network algorithm and the overlap degree of the mask drawn by the experts were used for calculation. Furthermore, the results drawn by the experts were used as the gold standard to obtain the segmentation effects of different neural networks. The average value $\bar{x}$ of the horizontal axis in the $3 \mathrm{D}$ ultrasound scan route drawn by the experts was used as the $x$ axis, and the vertical axis of the scan route was as the $y$ axis so as to establish a coordinate system. Thus, the standard error between the scan route of the neural network algorithm and the scan route drawn by the experts manually could be expressed by the following equation:

$$
\sigma=\sqrt{\frac{1}{l} \sum_{i}^{l}\left(y_{i}-\widehat{y}_{i}\right)^{2} .}
$$

In equation (14), $\sigma$ stands for the standard error, $l$ represents the number of ultrasound image columns, $y_{i}$ expresses the value corresponding to the $i$-th column in the scan route obtained after segmentation by different neural network algorithms, and $\widehat{y}_{i}$ means the value corresponding to the $i$-th column in the scan route manually drawn by the experts. In order to compare the segmentation effect of OctU-Net, U-Net and FCN were introduced for comparative analysis.

2.6. Comparison on PA Value and MIoU Value. The values of $\mathrm{PA}$ and MIoU were adopted in this study to compare the segmentation accuracy of different algorithms. A fixed-size resolution was adopted for the ultrasound images used in this study, and the image size was $256 \times 256$. The calculation method of PA and MIoU values could be expressed by the following functions:

$$
\begin{aligned}
\mathrm{PA} & =\frac{P_{t}}{P_{s}} \times 100 \%, \\
\mathrm{MIoU} & =\frac{1}{n} \sum_{i=1}^{n} \frac{\operatorname{Real}_{i} \cap \mathrm{Pre}_{i}}{\operatorname{Real}_{i} \cup \operatorname{Pre}_{i}} \times 100 \% .
\end{aligned}
$$

In equations (15) and (16), $n$ represents the number of images, Real $_{i}$ stands for the real labeled area, and Pre $_{i}$ means the predicted image area.

2.7. Statistical Methods. SPSS 19.0 statistical software was applied to process the data of this study. Measurement data were expressed as $\bar{x}$, and the mean standard deviation was represented by $\bar{x} \pm s$. Two independent sample $t$-tests were used for comparison between groups, count data were expressed as $n(\%)$, and $\chi^{2}$ was employed to test. $P<0.05$ meant that the difference was statistically substantial.

\section{Experimental Results}

3.1. Comparison on Recognition Accuracy of the Three Algorithms. 3D ultrasound examinations were performed on 3,300 pregnant women who participated in this study, and 2 ultrasound experts made judgments based on fetal ultrasound images. A total of 24 cases with spina bifida 
malformation were detected in the 3D ultrasound examinations. The 24 pregnant women were confirmed as fetal spina bifida during labor induction or after delivery in hospital. The diagnosis by ultrasound experts was correct, and the diagnosis accuracy rate was $100 \%$. Then, FCN, U-Net, and Oct-U-Net were adopted to judge the ultrasound images of all pregnant women, and the recall rate and precise rate of different algorithms were calculated. By comparing and analyzing the data, it was found that the recall rates and precise rates of FCN were 0.66 and 0.72 , respectively; the rates of U-Net were 0.73 and 0.75 , respectively; and those of Oct-U-Net were 0.93 and 0.96 , respectively (Figures 4 and 5). The recall rate and precise rate of Oct-U-Net were compared with those of FCN and U-Net, and the difference was statistically marked $(P<0.05)$. Thus, the segmentation effect of Oct-U-Net was superior to that of FCN and U-Net, and its diagnosis for fetal spina bifida was more accurate.

3.2. Analysis on the Segmentation Effects of the Three Algorithms. Different algorithms were used to identify and segment the 3D ultrasound images of pregnant women participating in this study and compare them with the results manually drawn by radiologists, as shown in Figures 6-8 (only ultrasound images diagnosed with fetal spina bifida were displayed). It could be concluded that although the FCN algorithm had fewer wrong segmentation results, the segmentation contour ended prematurely; the U-Net algorithm was affected by the scan path, and the segmentation contour was more accurate than the FCN algorithm; compared with the previous two algorithms, the Oct-U-Net algorithm segmentation contour was accurate, and the segmentation effect was ideal.

3.3. Comparison on Average Standard Errors of Prediction Results of the Three Algorithms. By comparing with the standard errors between the scan routes segmented by different neural network algorithms and the routes manually marked by experts, Oct-U-Net adopted in this study obtained more accurate segmentation results. The average standard error of Oct-U-Net, U-Net, and FCN was 4.1243, 12.8291 , and 14.1688 in turn. It revealed that the segmentation effect of Oct-U-Net was more accurate in contrast to that of U-Net and FCN. The segmentation results of different algorithms were compared, finding that the average error of FCN was higher but there were fewer wrong segmentation results because the FCN algorithm segmented images directly obtained from the upsampling layer (Figure 9). Although the segmented spine contour of U-Net was more accurate, there were too many similar regions in the ultrasound images, which affected the acquisition of the scan path of U-Net, resulting in a low accuracy of the U-Net scan path.

3.4. Analysis on PA and MIoU Values of the Three Algorithms. In order to further verify the effect of Oct-U-Net applied in the ultrasound images of fetal spina bifida, the PA and MIoU values were introduced for analysis and verification based on

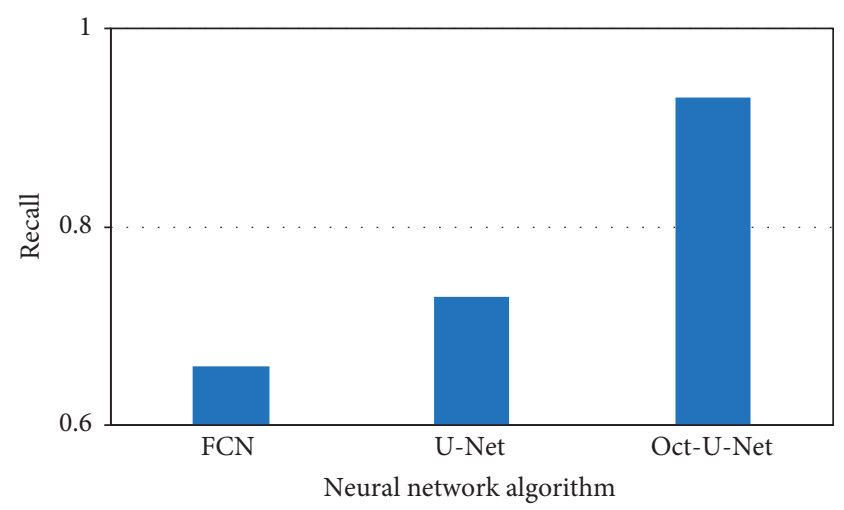

FIgURE 4: Comparison on the recall rates of different algorithms.

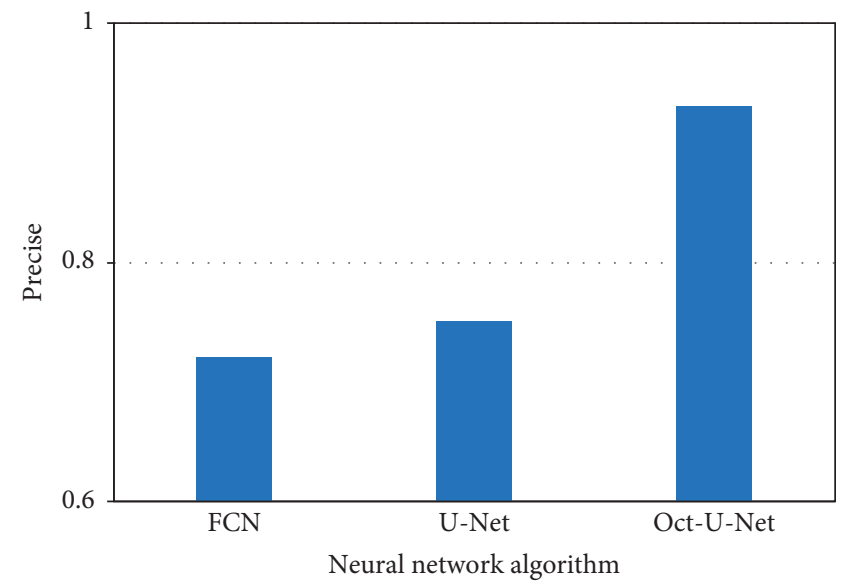

Figure 5: Comparison on the precise rates of different algorithms.

the segmentation results in Figures 6-8. The PA and MIoU values reflected the accuracy of different algorithms for identifying and segmenting ultrasound images of fetal spina bifida to a certain extent. The larger the PA value proving that the coverage of the algorithm segmentation area and the real area was greater and the MIoU value was larger. It indicated that the algorithm segmentation area was closer to the real area. In this study, the fetal ultrasound images of 24 cases with spina bifida deformation were used as the data set, and the area manually marked by ultrasound experts was used as the gold standard to evaluate FCN, U-Net, and OctU-Net. The results showed that the PA and MIoU values of the area manually marked by the experts were defined as 1 . Besides, the average PA and MIoU values of FCN were 0.766 and 0.798 , respectively; the average PA and MIoU values of U-Net were 0.803 and 0.815 in sequence; and the average PA and MIoU values of Oct-U-Net were 0.949 and 0.917 in turn. Therefore, it indicated that Oct-U-Net used in this study had a better segmentation effect compared with FCN and U-Net (Figures 10 and 11).

3.5. Comparison on Average Running Time of the Three Algorithms. When using different algorithms to diagnose fetal spina bifida ultrasound images, the running time of different algorithms was counted, as shown in Figure 12. The 


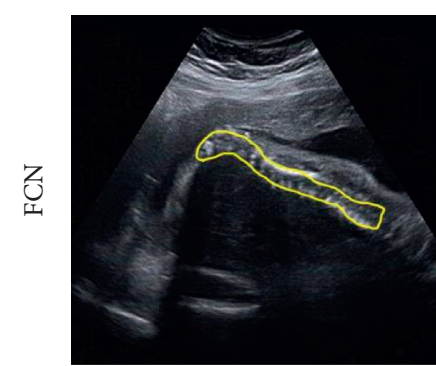

(a)

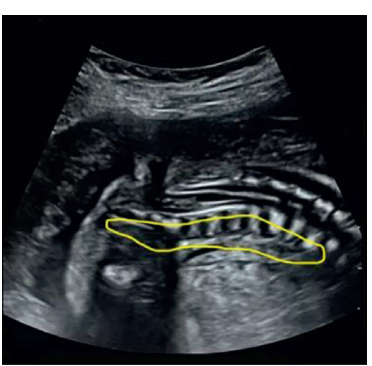

(b)

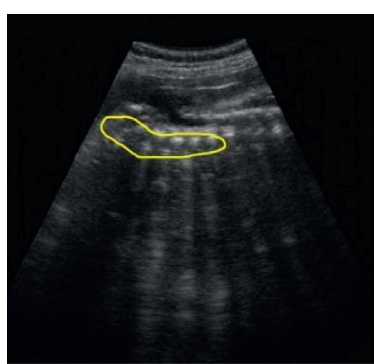

(c)

Figure 6: The segmentation effects of FCN.

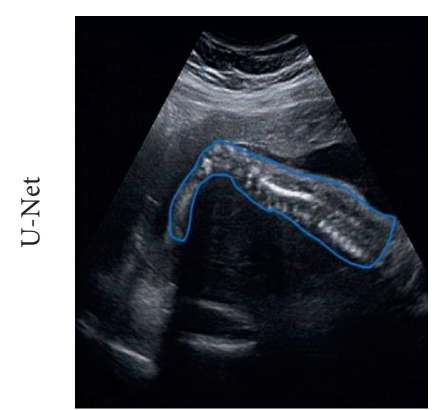

(a)

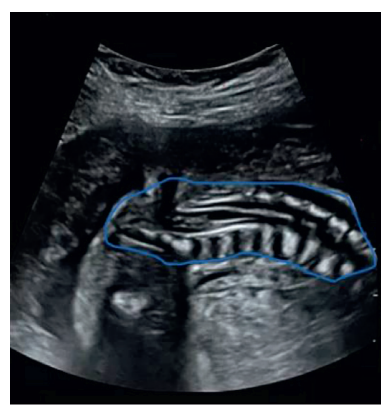

(b)

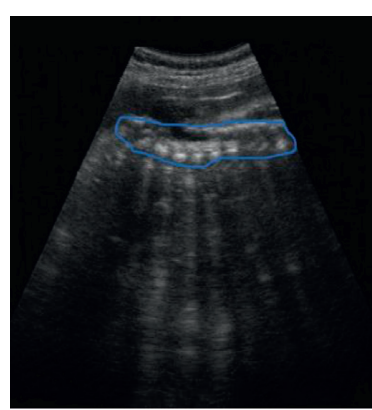

(c)

FIgURE 7: The segmentation effects of U-Net.

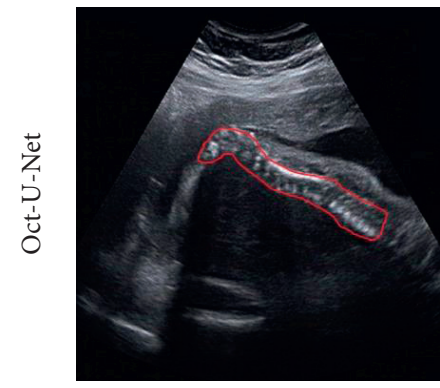

(a)

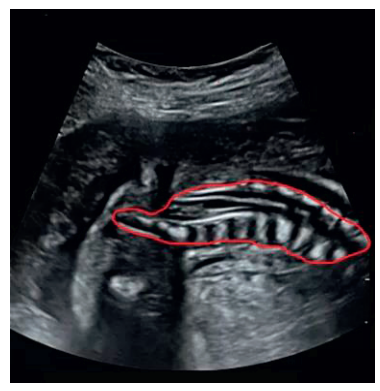

(b)

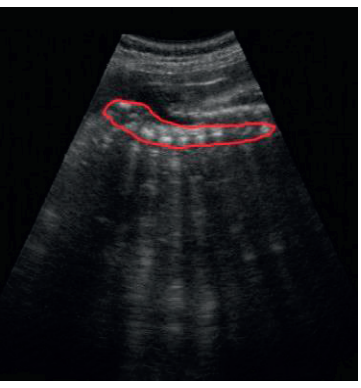

(c)

Figure 8: The segmentation effect of Oct-U-Net.

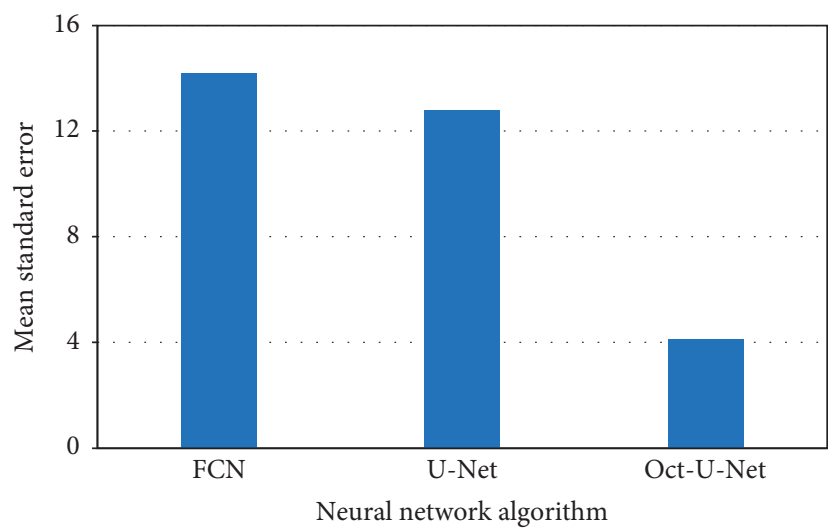

Figure 9: The average standard error analysis of different neural network algorithms. 


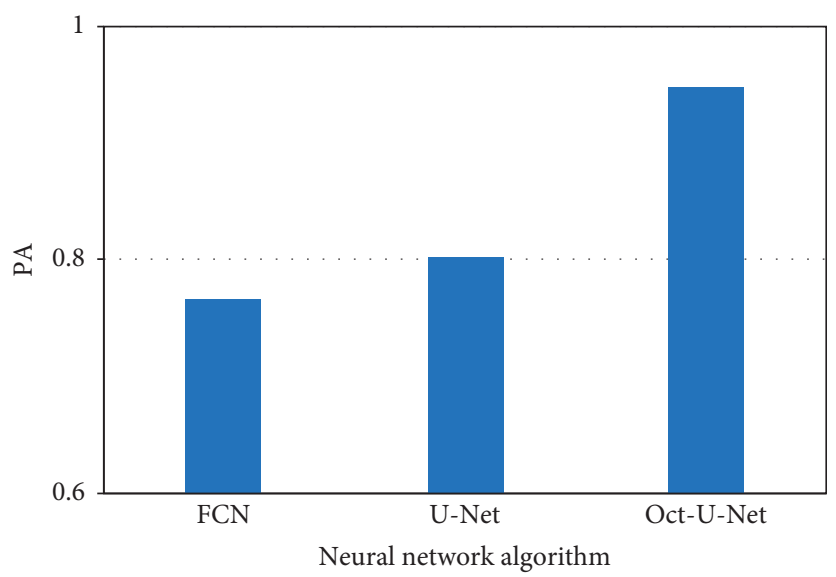

Figure 10: Comparison on MIoU values of different algorithms.

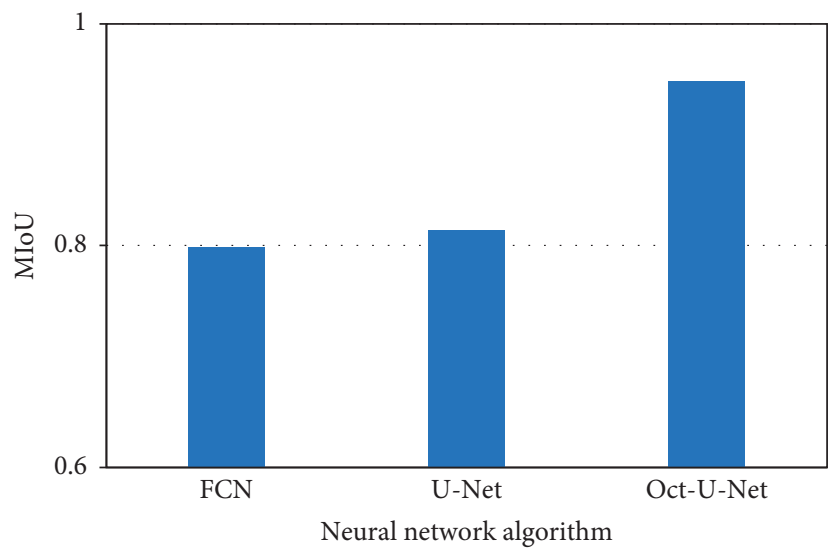

FIGURE 11: Comparison on MIoU values of different algorithms.

average running time of FCN was 16.14 seconds, the average running time of $\mathrm{U}-\mathrm{Net}$ was 22.33 seconds, and the average running time of Oct-U-Net was 12.15 seconds. It was found that the calculation time was greatly reduced because Oct-UNet replaced the two-layer convolution of U-Net with an Octave convolution. The running time of FCN was also less than that of U-Net due to its output images obtained directly from the upsampling layer. Thus, it disclosed that compared with FCN and U-Net, Oct-U-Net applied in this study effectively improved the segmentation speed of the ultrasound images of fetal spina bifida.

\section{Discussion}

Fetal spina bifida is a clinically common fetal congenital malformation. The mortality and disability of the fetus with spina bifida are very high, which will undoubtedly cause great pressure on the family. Therefore, ultrasound examination during pregnancy and timely termination of pregnancy according to the condition of spina bifida was the currently common treatment method. In order to better identify the images of fetal spina bifida, reduce the pressure of doctors to read the images, and improve the accuracy of diagnosis, U-Net was improved in this study and the twolayer convolution of U-Net was replaced with an Octave convolution so as to obtain the new Oct-U-Net neural network. Moreover, FCN and U-Net were introduced to compare with Oct-U-Net. In this study, 3,300 pregnant women were selected as the research objects, who received ultrasound examinations. Then, they were detected by $3 \mathrm{D}$ ultrasound, and whether the fetus had spina bifida was analyzed. The results of manual diagnosis by experts were used as the gold standard. Different algorithms were adopted to analyze and diagnose the $3 \mathrm{D}$ ultrasound images of pregnant women, and the recall and precise rates were employed to evaluate the accuracy of different algorithms. It showed that the recall and precise rates of Oct-U-Net applied in this study were 0.93 and 0.96 , respectively, which were greater than those of FCN and U-Net, and the difference was statistically obvious $(P<0.05)$. The standard errors between the three algorithms and the routes manually marked by experts were compared, indicating that the average standard error of Oct-U-Net was only 4.1243, which was much smaller than that of FCN and U-Net $(P<0.05)$. The segmentation accuracy of different algorithms was analyzed by $\mathrm{PA}$ and $\mathrm{MIoU}$ values, suggesting that the average PA and MIoU values of Oct-U-Net were 0.949 and 0.917 , respectively. In addition, both indicators were greater than FCN and U-Net, with a statistically marked difference $(P<0.05)$. The above results all revealed 


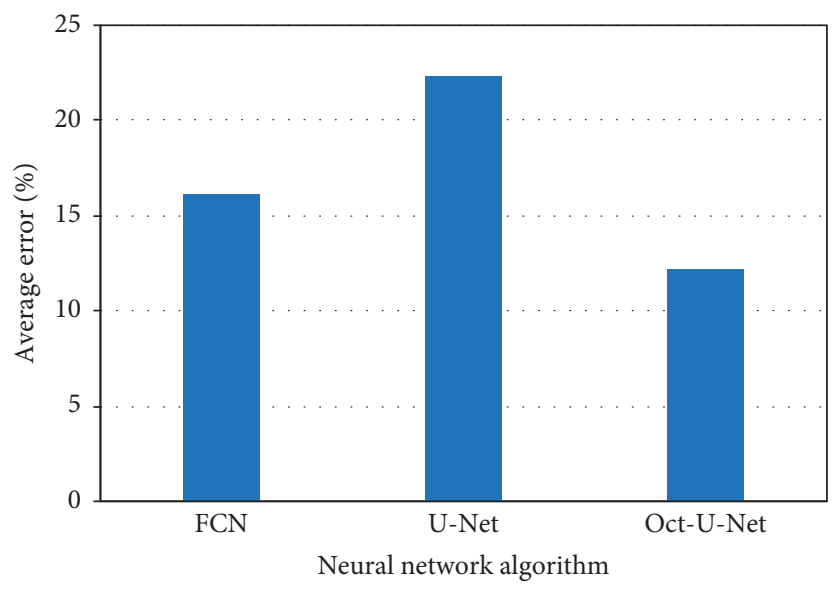

Figure 12: Comparison on running time of different algorithms.

that Oct-U-Net had excellent 3D ultrasound image segmentation and spina bifida recognition. This is similar to the results of Cengizler et al. [20]. Finally, it was found that the average running time of Oct-U-Net was 12.15 seconds, which was less than FCN and U-Net $(P<0.05)$ through a comparative analysis of the running time of the three algorithms. Therefore, Oct-U-Net could effectively shorten the segmentation time on the basis of promoting the segmentation accuracy.

\section{Conclusion}

The effect of Oct-U-Net on pregnant women's 3D ultrasound image segmentation was applied in this study to diagnose fetal spina bifida. The results showed that Oct-UNet could more accurately identify fetal spina bifida. Through analysis and verification, it was found that Oct-UNet had high recognition accuracy, good segmentation accuracy, and short running time. This algorithm has a good application prospect in the $3 \mathrm{D}$ ultrasound images for diagnosis of fetal spina bifida. This convolutional neural network algorithm is worthy of clinical application. The results of this study can provide a theoretical basis for the diagnosis of fetal spina bifida. However, the number of cases in this study is small, and the type of fetal spina bifida has not been subdivided. These deficiencies need to be explored more thoroughly with more clinical data in future work.

\section{Data Availability}

The data used to support the findings of this study are available from the corresponding author upon request.

\section{Conflicts of Interest}

The authors declare that they have no conflicts of interest.

\section{Authors' Contributions}

Lei Chen and Yingying Tian contributed equally to this work.

\section{References}

[1] Y.X. Sun, "Three dimensional ultrasound imaging technology in the diagnosis of fetal spina bifida," China Health Standard Management, vol. 20, no. 4, pp. 62-63, 2015.

[2] F. L. Ma, "The application of conventional ultrasonography combined with three-dimensional ultrasound in diagnosis of fetal spina bifida during early pregnancy," Diet Health, vol. 5, no. 28, pp. 264-265, 2018.

[3] T. Levin-Decanini, A. Houtrow, and A. Katz, "The evolution of spina bifida treatment through a biomedical ethics lens," HEC Forum, vol. 29, no. 3, pp. 197-211, 2017.

[4] C. Zhu, Y. Y. Ren, Y. L. Yan et al., "The value of prenatal ultrasonic diagnosis of fetal open and closed spina bifida," Fudan University Journal of Medical Sciences, vol. 43, no. 2, pp. 195-200, 2016.

[5] L. Mazzone, U. Moehrlen, B. Casanova et al., "Open spina bifida: why not fetal surgery?" Fetal Diagnosis and Therapy, vol. 45, no. 6, pp. 430-434, 2019.

[6] N. Prodan, M. Hoopmann, J. Sonek et al., "Fetal profile in fetuses with open spina bifida," Archives of Gynecology and Obstetrics, vol. 301, no. 5, pp. 1167-1171, 2020.

[7] X. T. Tang, "Ultrasound diagnosis value of open cleft spine and closed spina bifida," Journal of Imaging Research and Medical Applications, vol. 3, no. 6, pp. 41-43, 2019.

[8] W. Sepulveda, A. E. Wong, and F. Sepulveda, "Prenatal diagnosis of spina bifida: from intracranial translucency to intrauterine surgery," Child', vol. 33, no. 7, pp. 1083-1099, 2017.

[9] F. G. Sileo, P. Pateisky, J. Curado, K Evans, S Hettige, and $\mathrm{B}$ Thilaganathan, "Long-term neuroimaging and neurological outcome of fetal spina bifida aperta after postnatal surgical repair," Ultrasound in Obstetrics \& Gynecology : The Official Journal of the International Society of Ultrasound in Obstetrics and Gynecology, vol. 53, no. 3, pp. 309-313, 2019.

[10] M. Liu, Y. Liu, Z.-h. Li, and D. Yu, "Screening for fetal spina bifida aperta by the ultrasound and intracranial translucency examinations at 11-13(+6) Weeks of gestation," Cell Biochemistry and Biophysics, vol. 72, no. 2, pp. 439-441, 2015.

[11] H. Tang, H. L. Yao, R. H. Liang et al., "Comparative study of real-time three-dimensional ultrasound and two-dimensional ultrasound of fetal surface deformity and spinal deformity in second trimester," China Medicine and Pharmacy, vol. 6, no. 3, pp. 186-188, 2016. 
[12] E. B. Sha, C. L. Jing, L. Han, and Z. Gao, "Spina bifida occulta in prenatal ultrasound scan," Chinese Journal of Preventive Medicine, vol. 20, no. 3, pp. 190-195, 2017.

[13] S. Y. Tang, J. F. Xing, and M. Yang, "New method for medical image segmentation based on bp neural network," Computer Science, vol. 44, no. z1, pp. 240-243, 2017.

[14] Ç. Cengizler, M. K. Ün, and S. Büyükkurt, "A nature-inspired search space reduction technique for spine identification on ultrasound samples of spina bifida cases," Scientific Reports, vol. 10 , no. 1 , p. $9280,2020$.

[15] K. Zhu, Z. L. Fu, and X. Q. Chen, "Left ventricular segmentation method of ultrasound image based on convolutional neural network," Journal of Computer Applications, vol. 39, no. 7, pp. 2121-2124, 2019.

[16] B. Y. Tian, Y. Cheng, Y. H. Cai et al., "Ultrasound image segmentation for median nerves based on improved U-Net deep network," Process Automation Instrumentation, vol. 41, no. 8, pp. 36-41, 2020.

[17] Y. D. Li, Z. B. Hao, and H. Lei, "Survey of convolutional neural network," Journal of Computer Applications, vol. 36, no. 9, pp. 2508-2515, 2016.

[18] W. J. Liu, X. J. Liang, and H. C. Qu, "Learning performance of convolutional neural networks with different pooling models," Journal of Image and Graphics, vol. 21, no. 9, pp. 1178-1190, 2016.

[19] M. Z. Alom, C. Yakopcic, M. Hasan, T. M Taha, and V. K Asari, "Recurrent residual U-Net for medical image segmentation," Journal of Medical Imaging, vol. 6, no. 1, Article ID 014006, 2019.

[20] C. Cengizler, M. Kerem Ün, and S. Buyukkurt, "A novel evolutionary method for spine detection in ultrasound samples of spina bifida cases," Computer Methods and Programs in Biomedicine, vol. 198, Article ID 105787, 2021. 